\title{
Acanthamoeba Keratitis- Camouflage Entity in Eastern Nepal: A Case Series
}

\author{
- Sanjay Kumar Singh ${ }^{1} \bullet K^{2}$ arthik Ambur ${ }^{1} \bullet$ Prija Poudyal $^{1} \bullet$ Sagun Malla ${ }^{1} \bullet$ Amit Rajbanshi $^{1}$
}

Submitted 12 May 2021

$\risingdotseq$ Prija Poudyal

kgktssbh@gmail.com

iD https://orcid.org/0000-0003-4192-9791

${ }^{1}$ Biratnagar Eye Hospital, Biratnagar Morang, Nepal.

\section{Citation}

"Singh SK, Ambur K, Poudyal P, Malla S, Rajbanshi A. Acanthamoeba keratitiscamouflage entity in eastern Nepal: A case series. JBPKIHS. 2021;4(1):59-63.

doi https://doi.org/10.3126/jbpkihs.v4i1.37071

\section{(c) (i)}

This work is licensed under a Creative Commons AttributionNonCommercial 4.0 International License.
Accepted 12 June 2021

Published June 302021

Acanthamoeba keratitis is a sight-threatening corneal infection and is a growing clinical problem in the world. Though Acanthamoeba keratitis is considered uncommon and rarely reported in Nepal, we encountered six cases in 2019. All patients had redness, photophobia, decreased vision, and pain with ring infiltrate. Ten percent potassium hydroxide mount revealed Acanthamoeba cyst in all cases. Non-nutrient agar overladen with Escherichia coli revealed feeding tracks and Polymerase Chain Reaction revealed T4 genotype Acanthamoeba in four cases. Amoebicidal treatment was started with chlorhexidine 0.02\% eye drop half-hourly and supplementary treatment included moxifloxacin eye drop, a combination of polymyxin B sulfate, neomycin sulfate, and bacitracin eye ointment. After treatment, one patient had the best-corrected visual acuity of 6/9 while others had a visual outcome of hand movement. A high level of clinical suspicion and wet mount examination of specimen from infected corneal tissue are essential to aid in rapid diagnosis.

Keywords: Acanthamoeba keratitis, Cornea, Polymerase Chain Reaction, Nepal

\section{Declarations}

Ethics approval and consent to participate: Not applicable. However, Institutional Research Committee was informed about the case report. Consent for publication: Informed consent obtained from patients for publication of identifying features.

Availability of data and materials: All data generated or analyzed during this study are included in this published article and the patients' files can be presented if required.

Competing interest: None

Funding: None

Authors' contributions: SKS: ophthalmic care, literature review, manuscript preparation and editing. KA: ophthalmic care, literature review, data collection. PP: laboratory investigations, literature review, manuscript preparation. SM: ophthal- mic care, literature review, data collection. AR: laboratory investigation, literature review.All authors read and approved the final manuscript.

Acknowledgement: We would like to acknowledge Dr. Ibne Karim M. Ali, $\mathrm{PhD}$, and Mr. Shantanu Roy, MSc. Microbiology, Division of Foodborne, Waterborne and Environmental Diseases, Center for Disease Control and Prevention $(C D C)$, Atlanta for providing the real time PCR reports and for the immense support and cooperation. 
$A$ canthamoeba species are free-living protozoans and have been isolated from air, water, and soil $[1,2]$. It was first reported in 1973 [3]. Various risk factors are responsible for its causation including the use of contact lens in developed countries [4-6], and trauma or use of contaminated water in developing countries [3, 7-9]. Acanthamoeba adheres to the corneal epithelium with the help of a protein and then produce pathogenic proteases that degrade the basement membrane and cause cytolysis and apoptosis of cornea cellular elements and finally lead to the dissolution of the corneal stroma [10]. Acanthamoeba keratitis is often misdiagnosed as it shares similar symptoms and signs as that of herpetic, fungal, or bacterial keratitis. So, accompanying diagnostic tests with microscopy, culture, and Polymerase Chain Reaction (PCR) are highly supportive.

\section{CASES}

$\mathrm{T}$

This retrospective case series included all the Acanthamoeba keratitis cases encountered in the year 2019 at Biratnagar Eye Hospital, Biratnagar, Nepal. All six Acanthamoeba patients were from agricultural background and none of the patients wore contact lenses. Four patients had a history of trauma with vegetative matters. Patients presented late to the eye hospital and the duration of onset of symptoms to presentation to our hospital (mean $\pm \mathrm{SD}$ ) was $19 \pm 7.78$ days. Five patients had best corrected visual acuity (BCVA) of hand movement to the perception of light vision and one had 2/60 in the affected eye at the time of presentation (Table 1).

All patients had redness, photophobia, and decreased vision. Severe excruciating pain was the main complaint in two patients (Case 1 and 3, Table 1). Ring infiltrate was the hallmark in all the patients (Fig. 1). The size of the corneal ulcer was $>6 \mathrm{~mm}$ in four patients (Table 2).

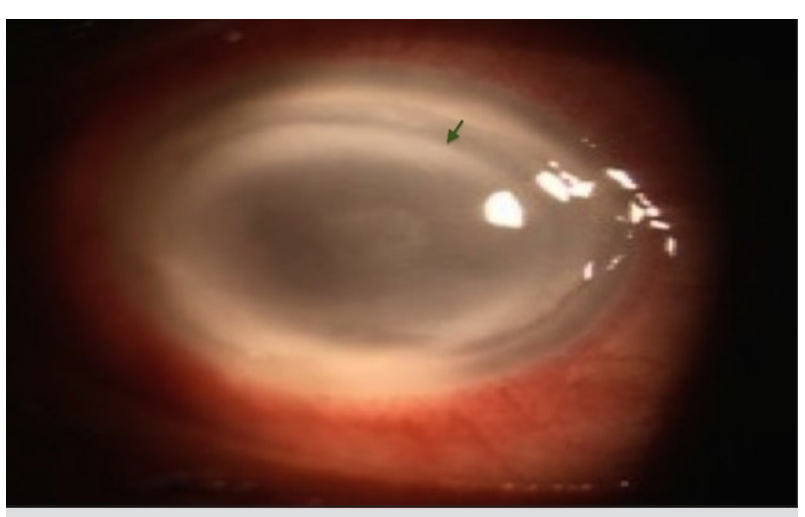

Figure 1: Ring infiltrate appreciated in an affected eye

\section{MICROBIOLOGICAL LABORATORY WORK-UP}

All patients underwent corneal scraping with a 15-degree Bard-Parker blade and scraped tissue was sent for gram stain and $10 \%$ potassium hydroxide $(\mathrm{KOH})$ mount respectively. It was also inoculated in blood agar, chocolate agar, and Sabouraud dextrose agar. If the Acanthamoeba cysts were identified in either gram stain or $\mathrm{KOH}$ mount, then scraping was further inoculated in non-nutrient agar overladen with Escherichia coli.

Double-walled cysts of Acanthamoeba were noted on $\mathrm{KOH}$ mount (Fig. 2a) in the first scraping in four cases whereas only in the second scraping that was done after 3 days in two cases (Table 3). The second scrapings were done as there was high clinical suspicion suggesting Acanthamoeba. Gram stain revealed Acanthamoeba cyst (Fig. 2b) in one of the cases (Case 5). The culture of the first four cases in the non-nutrient agar with Escherichia coli showed clear/ feeding tracks along the lawn of Escherichia coli which signified the presence of migrating trophozoites that feed on the bacilli (Fig. 2c). Cysts isolated from culture showed inner polygonal and outer wrinkled wall (Fig. 3a) which was further highlighted with Giemsa stain (Fig. 3b). The

\begin{tabular}{|c|c|c|c|c|c|}
\hline Case No & Age/ Sex & Involved eye & Vegetative trauma & Vision at presentation & $\begin{array}{l}\text { Onset to presentation } \\
\text { duration (days) }\end{array}$ \\
\hline 1 & $47 / \mathrm{F}$ & Left & Yes & $\mathrm{HMCF}$ & 15 \\
\hline 2 & $28 / M$ & Right & No & POL* & 30 \\
\hline 3 & $32 / M$ & Right & Yes & $2 / 60$ & 10 \\
\hline 4 & $32 / \mathrm{F}$ & Left & No & $\mathrm{POL}^{*}$ & 25 \\
\hline 5 & $33 / M$ & Right & Yes & $\mathrm{HMCF}$ & 20 \\
\hline 6 & $58 / M$ & Right & Yes & $\mathrm{HMCF}$ & 12 \\
\hline
\end{tabular}

HMCF: Hand movement close to face, $P O L^{*}$ : perception of light, projection of rays accurate in all quadrants 


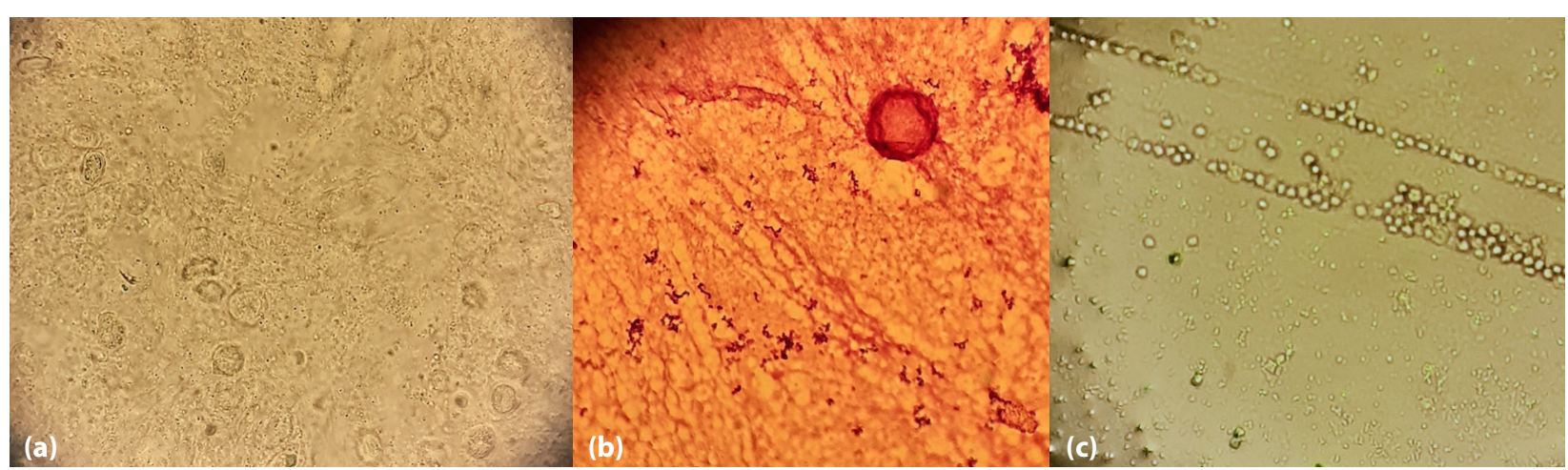

Figure 2: Acanthamoeba cyst (a) seen under $10 \% \mathrm{KOH}$ mount $(10 \mathrm{X}) \quad$ (b) observed with Gram stain (100X) $($ c) amoebal feeding tracks appreciated along the lawn of E.coli (10X)
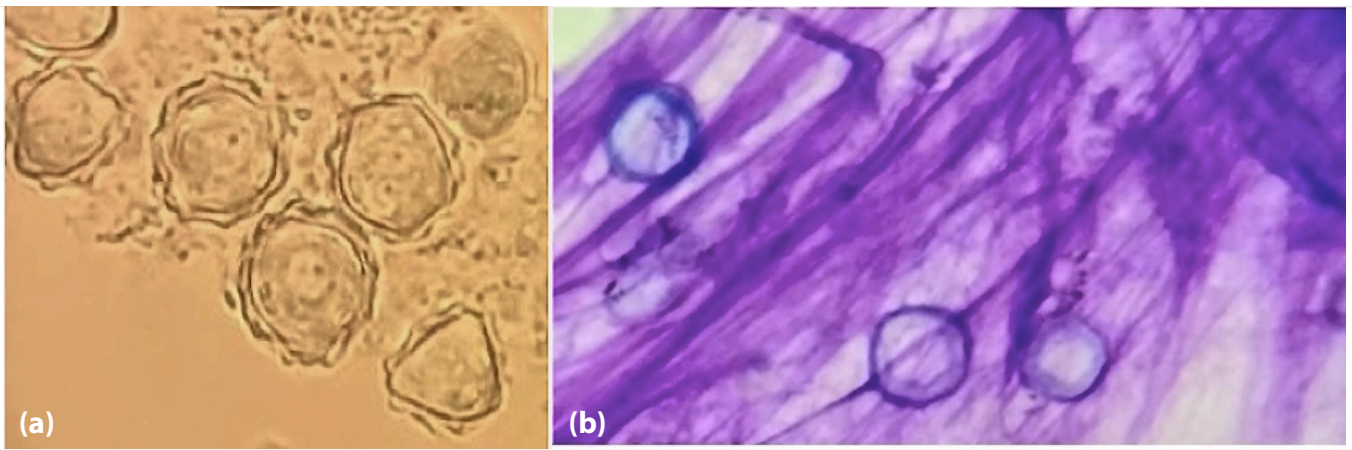

Figure 3: Acanthamoeba cyst (a) isolated from culture plates revealing inner polygonal and outer wrinkled wall (100X) (b) further highlighted with Giemsa stain(40X)

blood agar, chocolate agar, and Sabouraud dextrose agar cultures were negative in all cases.

PCR testing facility was not available anywhere in Nepal for Acanthamoeba at that time, so communication was made with the Centre for Disease Control and Prevention, USA and the samples of the initial four patients were sent in a special carrier media, as per their instructions. All first four samples were Acanthamoe$b a$ positive by a diagnostic real-time PCR (Table 3). These samples were genotyped successfully and belong to genotype $\mathrm{T}_{4}$. Two of the strains appeared identi- cal in Sanger sequences to each other, while the other two were distinct. Three of these samples showed the most resemblance with the Acanthamoeba culbertsoni. Samples of the last two patients, who lost to follow-up, could not be sent.

\section{TREATMENT}

In this hospital, five patients except one (Case 3) received amoebicidal treatment after confirmation of clinical suspicion with direct microscopy reports. Out of these five patients, three were treated with antifun-

\begin{tabular}{llll}
\multicolumn{2}{l}{ Table $2:$ Clinical findings on ophthalmic examination } \\
\hline \begin{tabular}{llll} 
Case & Ulcer location & Size of ulcer $(\mathrm{mm})$ & Hypopyon \\
No. & & & \\
\hline I & Central + paracentral & 7.0 & Present \\
2 & Central + paracentral & 7.0 & Present \\
3 & Paracentral & 4.0 & Absent \\
4 & Central + paracentral & 5.0 & Absent \\
5 & Central + paracentral & 6.5 & Absent \\
6 & Central + paracentral & 8.0 & Present \\
\hline
\end{tabular}
\end{tabular}


Table 3: Microbiological and PCR findings from the scraped samples

\begin{tabular}{|c|c|c|c|c|c|c|}
\hline \multirow{3}{*}{ Case No. } & \multicolumn{4}{|c|}{ Acanthamoeba like cyst detected } & \multirow{3}{*}{$\begin{array}{l}\text { Culture* } \\
\text { positive on day }\end{array}$} & \multirow{3}{*}{ PCR } \\
\hline & \multicolumn{2}{|l|}{ I st $_{\text {scraping }}$} & \multicolumn{2}{|l|}{$2^{\text {nd }}$ Scraping } & & \\
\hline & Gram staining & $\mathrm{KOH}$ & Gram staining & $\mathrm{KOH}$ & & \\
\hline I & None & None & None & Yes & 4 & Positive \\
\hline 2 & None & Yes & ND & ND & 5 & Positive \\
\hline 3 & None & None & None & Yes & 5 & Positive \\
\hline 4 & None & Yes & ND & ND & 7 & Positive \\
\hline 5 & Yes & Yes & ND & ND & ND & ND \\
\hline 6 & None & Yes & ND & ND & ND & ND \\
\hline
\end{tabular}

ND: Not done, $\mathrm{KOH}: 10 \%$ potassium hydroxide, $P C R$ : Polymerase Chain Reaction. ${ }^{*}$ Non- nutrient agar with Escherichia coli.

gal and antibiotics while two were treated with antiviral and antibiotics elsewhere before they approached us with a history of progression of infection and no alleviation of symptoms. Amoebicidal treatment was started with chlorhexidine $0.02 \%$ eye drop. Initially, the drug was started on a half-hourly basis and the dose was reduced after 3 days to an hourly basis, subsequently, it was tapered to 2 hourly after one week. Supplementary treatment included treatment with moxifloxacin eye drop 6 times a day, a combination of polymyxin B sulfate, neomycin sulfate, and bacitracin ointment at bedtime and atropine eye drop 3 times a day. Case no 3 was continued on antifungal (natamycin eye drop $5 \%$ half hourly and fluconazole eye drop $0.3 \%$ half hourly) and antibiotic (moxifloxacin eye drop $0.5 \%$ half hourly) as the patient was already receiving the medication before he was diagnosed in our hospital and the scarring had started around the ulcer despite thin cornea. He underwent cyanoacrylate glue application along with the bandaged contact lens. Gradually eye drops were tapered in all patients.

All patients showed early response to medication with a dramatic reduction in pain within one week as noted subjectively. Cases 1, 2 and 4 had healing with early corneal scarring within 2-3 weeks. One patient (Case 3) had gained BCVA of 6/9 whereas the remaining 3 patients had a final vision of hand movement. Case 5 and 6 visited another eye hospital to seek a second opinion and were lost to follow-up.

\section{DISCUSSION}

$\mathrm{U}$ nlike the reports of Acanthamoeba keratitis from the developed world [4-6], none of our patients wore contact lens, and all were farmers. Similar findings of higher association of Acanthamoeba keratitis with a his- tory of trauma due to vegetative matter or other foreign body have been reported from other developing countries [3, 7-9].

In this series, excessive pain disproportionate to sign was complained by two patients (case no 1 and 3) while the other four patients complained of little pain. Ring infiltrate, a diagnostic sign for Acanthamoeba keratitis was noticed in all patients. Similar clinical features were described in earlier reports [3, 7].

In this case series, the sensitivity of $\mathrm{KOH}$ was higher than gram-stained smears in the detection of Acanthamoeba, as the cysts were easily identified in all six cases using $\mathrm{KOH}$ mount compared to one case using gram stain. Several other studies from India have reported superiority of $\mathrm{KOH}$ wet mount preparation for the diagnosis of Acanthamoeba keratitis [3, 7]. Acanthamoeba cysts with trophozoites were identified on non-nutrient agar overladen with Escherichia coli for initial four patients and PCR reporting was also reported to be positive in these patients.

All patients showed a clinical response to treatment. The pain was significantly reduced in all patients after starting medication and this could be due to the effect of amoebicidal and atropine eye drops. Two patients preferred to have a second opinion so they could not be followed up. Only one patient had BCVA of 6/9 and others had visual outcome of hand movement. Centrally located ulcer, size of the ulcer $>6 \mathrm{~mm}$, and visual acuity hand movement/ perception of light at the time of presentation were associated with poor outcome.

Despite having one of the highest reported incidences of corneal ulcer in the world [11, 12], there are very few published reports of Acanthamoeba keratitis from Nepal [13]. It may be due to misdiagnosis relating to its simulation with bacterial, fungal, or viral infections. The diagnosis of Acanthamoeba keratitis should 
be considered in patients with symptoms of photophobia and disproportionate pain. The pathognomic sign is a radial pattern of perineural infiltrates [14]. Also, in the presence of clinical signs of Acanthamoeba keratitis, diagnostic tests should always be performed [15]. To the best of our knowledge, this happens to be the first report of Acanthamoeba keratitis with PCR verified results in our country.

\section{CONCLUSION}

canthamoeba keratitis is a growing clinical probIlem in developed as well as developing countries. A sound clinical suspicion and accompanied microbiological investigation is needed for the early diagnosis of this condition.

\section{References}

I. Stothard DR, Schroeder-Diedrich JM,Awwad MH, Gast RJ, Ledee $D R$, Rodriguez-Zaragoza $S$, et al. The evolutionary history of the genus Acanthamoeba and the identification of eight new 185 rRNA gene sequence types. J Eukaryot Microbiol. 1998;45(I):4554. DOI: I0.1 I II/j.1550-7408.1998.tb05068.x

2. Marciano-Cabral F, Cabral G.Acanthamoeba spp. as agents of disease in humans. Clinical Microbiology Reviews. 2003;16(2):273307. DOI: 10.1 I 28/cmr.16.2.273-307.2003

3. Bharathi JM, Srinivasan M, Ramakrishnan R, Meenakshi R, Padmavathy S, Lalitha PN.A study of spectrum of Acanthoemba keratitis: a three year study at a tertiary eye care referral center at South India. Indian Journal of Ophthalmology. 2007;55(I):37-42. DOI: I0.4I03/030I-4738.29493

4. Chin J, Young AL, Hui M, Jhanji V. Acanthamoeba keratitis: I0-year study at a tertiary eye care center in Hong Kong. Cont Lens Anterior Eye. 2015;38(2):99-I03. DOI: I0.1016/j.clae.20I4.II.I46

5. Lindsay RG, Watters G, Johnson R, Ormonde SE, Snibson GR. Acanthamoeba keratitis and contact lens wear. Clin Exp Optom. 2007;90(5):35 I-60. DOI: I0.1 I I I/j. I 444-0938.2007.00 I72.x

6. Stehr-Green JK, Bailey TM, Brandt FH, Carr JH, Bond WW, Visvesvara GS. Acanthamoeba keratitis in soft contact lens wearers. A case-control study. JAMA. 1987;258(I):57-60. PMID: 3586292

7. Sharma S, Srinivasan M, George C. Acanthamoeba keratitis in non-contact lens wearers. Arch Ophthalmol. 1990;108(5):676-8. DOI: 10.100I/archopht. 1990.01070070062035

8. Garg P, Kalra P, Joseph J. Non-contact lens related Acanthamoeba keratitis. Indian J Ophthalmol. 20I7;65(II):1079-86. DOI: 10.4103/ijo.IJO_826_17
9. Sharma S, Garg P, Rao GN. Patients characteristics, diagnosis and treatment of non-contact lens related Acanthamoeba keratitis. $\mathrm{Br}$ J Ophthalmol. 2000;84(I0): I I03-8. DOI: I 0. I I36/bjo.84. I0.I I03

10. Clarke DW, Niederkorn JY.The pathophysiology of Acanthamoeba keratitis. Trends Parasitol. 2006;22(4):175-80. DOI:10.1016/j. pt.2006.02.004

II. Upadhyay MP, Karmacharya PC, Koirala S, Shah DN, Shakya S, Shrestha JK, et al. The Bhaktapur eye study: ocular trauma and antibiotic prophylaxis for the prevention of corneal ulceration in Nepal. Br J Ophthalmol. 200I;85(4):388-92. DOI: 10.1/36/ bjo.85.4.388

12. Puri LR, Shrestha, GS. Microbial keratitis: A five years retrospective clinical study in tertiary eye hospital of eastern region of Nepal. Journal of Kathmandu Medical College. 2017;4(4): I 18-25. DOI: $10.3126 / j \mathrm{kmc} . v 4 \mathrm{i} 4.18252$

13. Sah R, Chaudhary M, Khadka S, Toledo R, Acosta L. Non- related contact lens coinfection with Acanthamoeba and Fusarium. Asian Pac JTrop Med. 2019;1 2:479-82. DOI: 10.4103/1995-7645.269909

14. Panwar P, Sharma K. Acanthamoeba keratitis-a diagnostic dilemma: a case report. Adv Ophthalmol Vis Syst. 2018;8(I):52-3. DOI: I0.I5406/aovs.2018.08.0026

15. Szentmary N, Daas L, Shi L, Laurik KL, Lepper S, Milioti G, et al. Acanthamoeba keratitis- Clinical signs, differential diagnosis and treatment. J Curr Ophthalmol. 2019;31(1):16-23. DOI: I0.1016/j. joco.2018.09.008 